\title{
Light of Planck-2015 on Noncanonical Inflation
}

\author{
Kh. Saaidi, ${ }^{1}$ A. Mohammadi, ${ }^{2}$ and T. Golanbari ${ }^{3}$ \\ ${ }^{1}$ Islamic Azad University, Sanandaj Branch, Pasdaran Street, P.O. Box 618, Sanandaj, Iran \\ ${ }^{2}$ Young Researchers and Elites Club, Sanandaj Branch, Islamic Azad University, Sanandaj, Iran \\ ${ }^{3}$ Department of Physics, Faculty of Science, University of Kurdistan, Pasdaran Street, Sanandaj 66177-15175, Iran
}

Correspondence should be addressed to Kh. Saaidi; ksaaidi@uok.ac.ir

Received 6 May 2015; Accepted 24 June 2015

Academic Editor: Rong-Gen Cai

Copyright (C) $2015 \mathrm{Kh}$. Saaidi et al. This is an open access article distributed under the Creative Commons Attribution License, which permits unrestricted use, distribution, and reproduction in any medium, provided the original work is properly cited. The publication of this article was funded by $\mathrm{SCOAP}^{3}$.

Slow-roll inflationary scenario is considered in noncanonical scalar field model supposing a power-law function for kinetic term and using two formalisms. In the first approach, the potential is picked out as a power-law function, that is, the most common approach in studying inflation. Hamilton-Jacobi approach is selected as the second formalism, so that the Hubble parameter is introduced as a function of scalar field instead of the potential. Employing the last observational data, the free parameters of the model are constrained, and the predicted form of the potential and attractor behavior of the model are studied in detail.

\section{Introduction}

Inflationary scenario is the best candidate for describing the very early times evolution of the universe, which could simply solve the problems of the standard hot big bang model. There are lots of observational data supporting this scenario [1]. The scenario was first proposed by American physicist, Guth, in order to solve the problem of standard hot big bang model [2]. So far, different model of inflationary cosmology has been introduced; however it might be said that the most interesting one is chaotic inflation, introduced by Linde in 1983 [3]. Based on this scenario, the universe in the very early times is dominated by a slowly varying homogenous scalar field and undergoes an exponential expansion in very short time.

The scenario has been constructed based on canonical scalar field; however recently the cosmological models of scalar field including noncanonical kinetic term have attracted scientists attention. The general form of its action is expressed by $\mathscr{L}_{\phi}=f(\phi) F(X)-V(\phi)$, where $X=\left(\nabla_{\mu} \phi \nabla^{\mu} \phi\right) / 2$ [4]. The case with $V(\phi)=0$ comes into a well-known model as $k$-essence. The main idea of $k$-essence comes from BornInfeld action of string theory $[5,6]$. The model is able to give some interesting results about dark energy [7-12]. In $[13,14]$, the model is applied as a possible way for inflation and describing early time evolution of the universe.
In the present work, we are going to take $f(\phi)=1$ and $V(\phi)$ as scalar field potential. In other works, we take a pure kinetic $k$-essence plus a potential term. This kind of model is known as noncanonical scalar field [15]. This case is another class of the general form which could be as important and interesting as $k$-essence model. The cosmological solution of the model has been studied in [15], where it was shown that it is possible to construct a unified model of dark matter and dark energy for a simple form of noncanonical kinetic term $F(X)$. The same case has been considered in [16] as well, in which they found that producing a unified model of dark matter and dark energy for a pure kinetic $k$-essence is very difficult. It seems that the noncanonical scalar field model has this ability to be an appropriate model of the universe evolution and has merit of consideration in more detail. Then, we were motivated to use noncanonical scalar field as a possible model for describing one example of the earliest universe evolution, namely, inflation.

There are two formalisms that cosmologists apply to study inflation, and in the present work, we are going to utilize both these approaches. The first formalism, and the most common and well-known one, relies on the potential. In this approach, we need to propose a specific form of the potential to be able to investigate the model in detail. In this regard, 
during this work, a familiar form of potential, as powerlaw, is picked out. The power-law potential, $\lambda \phi^{\alpha}$, has a huge contribution in inflationary scenario studies, which includes chaotic inflation. The two most known cases are $\alpha=4$ and $\alpha=2$. Recent observational results have shown that the $\lambda \phi^{4}$ stays outside of the joint $99.7 \%$ CL region in the $n_{s}-r$ plane. The quadratic potential model, $\alpha=2$, lies outside the joint 95\% CL region for Planck $+\mathrm{WP}+$ high-l for $N \leq 60 e$ folds. The potential models with $\alpha=1$ and $\alpha=2 / 3$ lie on the boundary of the joint 95\% CL region [17-19]. Another interesting formalism is known as Hamilton-Jacobi formalism, which relies on a specific form of the Hubble parameter in terms of scalar field, instead of introducing a potential [2024]. In comparison with the first formalism, Hamilton-Jacobi formalism includes some fascinating feature such as deducing a form of the potential, obtaining an exact solution for the scalar field. Although the formalism has got less attention than first formalism, its abilities in inflationary studies sound undeniable. Based on the presented argument, the later formalism stands in the center of our attention in this work.

The main goal of the work is to constrain the free parameters of the model utilizing the latest observational data released by Planck-2015 [19, 25]. Prediction of quantum perturbation is one of interesting features of inflationary scenario. The most important ones of these perturbations are scalar perturbation, which is seeds for large scale structure of the universe, and tensor perturbation, which is known as gravitational waves too [26-28]. The prediction is supported by huge amount of observational data. From Planck data, the amplitude of scalar perturbation is about $\ln \left(10^{10} A_{s}^{2}\right)=3.094$, and the scalar spectra index, which is equal to one for a scaleinvariant spectrum, is measured about $n_{s}=0.9645$ [19]. In contrast with scalar perturbation, Planck does not give an exact value for tensor-to-scalar ratio $r$; it just specifies an upper bound for this parameter as $r<0.10$ [19].

The paper is organized as the following: the general form of noncanonical scalar field equations, slow-rolling parameters, and the perturbation parameters are derived in Section 2. In Section 3, the problem is studied by using the first formalism, and appropriate values for the free parameters are presented. The second formalism is applied in Section 4, where, as well as constraining the parameters, the predicted potential and attractor behavior of the model are considered.

\section{Noncanonical Scalar Field}

To begin, we introduced the Lagrangian which is read as

$$
\mathcal{S}=\int d^{4} x \sqrt{-g}\left(\frac{M_{p}^{2}}{2} R+\mathscr{L}_{N}\right),
$$

where $R$ is Ricci scalar constructed from the metric $g_{\mu \nu}$ and $M_{p}$ is Planck mass. $\mathscr{L}_{N}$ is the Lagrangian of noncanonical scalar field which is defined as $\mathscr{L}_{N}=F(X)-V(\phi)$. The kinetic term of noncanonical scalar field is expressed by an arbitrary function $F(X)$, in which $X=-\left(g^{\mu \nu} \nabla_{\mu} \phi \nabla_{\nu} \phi\right) / 2$. Potential of scalar field is denoted by $V(\phi)$.
The observation shows that the universe is spatially flat. The common metric to describe such a universe is spatially flat FLRW metric. The dynamical equations are derived by taking variation of action with respect to independent variable as $g_{\mu \nu}$ and $\phi$. Substituting the metric in the field equations leads to well-known Friedmann equations as

$$
\begin{aligned}
H^{2} & =\frac{1}{3 M_{p}^{2}} \rho, \\
\dot{H} & =-\frac{1}{2 M_{p}^{2}}(\rho+p)
\end{aligned}
$$

in which $\rho$ and $p$ are, respectively, the energy density and pressure of noncanonical scalar field, expressed by

$$
\begin{aligned}
& \rho=2 X F_{X}-F+V(\phi), \\
& p=F-V(\phi) .
\end{aligned}
$$

Derivative of kinetic function $F(X)$ with respect to $X$ is denoted by $F_{X}$. And for the acceleration equation we have

$$
\frac{\ddot{a}}{a}=H^{2}+\dot{H}=-\frac{1}{3 M_{p}^{2}}\left(F+X F_{X}-V(\phi)\right) \text {. }
$$

To have a positive acceleration phase for the universe, one must have $V(\phi)>\left(F+X F_{X}\right)$. On the other hand, the equation of motion of noncanonical scalar field is obtained

$$
\left(2 X F_{X X}+F_{X}\right) \ddot{\phi}+3 F_{X} H \dot{\phi}+V^{\prime}(\phi)=0
$$

which is another expression of familiar conservation equation $\dot{\rho}+3 H(\rho+p)=0$, where $\rho$ and $p$ have been introduced in (3).

From now on, we take the kinetic term as a power-law function of $X$; namely, $F(X)=F_{0} X^{n}$. The constant $n$ is dimensionless, and $F_{0}$ is a constant whose dimension is fitted in a way to give $\left[M^{4}\right]$ for kinetic energy density $F(X)$. It could be easily checked that the case $n=1$ and $F_{0}=1$ comes to usual canonical scalar field model.

2.1. Noncanonical Inflation. Inflation is an era of the universe evolution where it stays in a positive accelerated phase and undergoes an extreme expansion. It is supposed that, in this era, the universe is dominated by an isotropic and homogeneous scalar field which causes quasi-de Sitter expansion. During this work, we assume that inflation happens due to a noncanonical inflation, and the general form of parameters is derived.

2.1.1. Slow-Roll Approximation. In order to have a quasi-de Sitter expansion, the rate of the Hubble parameter during a Hubble time should be much smaller than unity; in other words $\dot{H} / H^{2} \ll 1$ [29]. The same situation is assumed for $\dot{\phi}$, which states that the rate of time derivative of scalar field during a Hubble time should be much smaller than unity: $|\ddot{\phi}| / H|\dot{\phi}| \ll 1$ [29]. These two conditions are known as slowroll approximations. The first condition allows us to ignore the kinetic energy density of scalar field against the potential 
part in the Friedmann equation, and the second condition lets one ignore the term $\ddot{\phi}$ against the term $H \dot{\phi}$ in the wave equation. Corresponding to each slow-roll approximation there is a slow-roll parameter, given by [29]

$$
\begin{aligned}
& \epsilon_{H}=-\frac{\dot{H}}{H^{2}}, \\
& \eta_{H}=-\frac{|\ddot{\phi}|}{H|\dot{\phi}|} .
\end{aligned}
$$

Smallness of these two parameters during inflation shows that the scalar field slowly rolls down its potential and lets enough amount of inflation happen.

2.1.2. Perturbation. Inflationary models predict three kinds of perturbations as scalar, vector, and tensor perturbation. One of the main metric perturbations is scalar perturbation. Scalar fluctuations become seeds for cosmic microwave background (CMB) anisotropies or for large scale structure (LSS) formation. Therefore, by measuring the spectra of the CMB anisotropies and density distribution, the corresponding primordial perturbation could be determined. First, let us have a brief look at the scalar perturbation.

Consider only an arbitrary scalar perturbation to the background FLRW metric, which is expressed by [26-28, 30, 31]

$$
\begin{aligned}
d s^{2}= & -(1+2 A) d t^{2}-2 a^{2}(t) \nabla_{i} B d x^{i} d t \\
& +a^{2}(t)\left[(1-2 \psi) \delta_{i j}+2 \nabla_{i} \nabla_{j} E\right] d x^{i} d x^{j}
\end{aligned}
$$

$\delta_{i j}$ is background spatial metric and $\nabla_{i}$ is the covariant derivative with respect to this metric. The intrinsic curvature of the spatial hypersurface is expressed by the perturbation parameter $\psi$ as ${ }^{3} R=4 \nabla^{2} \psi / a^{2}$, where $\psi$ is usually named the curvature perturbation [31].

Inserting this metric in the main field equations leads one to the scalar perturbation, which are expressed in [26$28,30-33]$. In this order, we follow [30], which has considered the perturbation of generalized gravity, including our model. After some routine algebraic calculation, the results for scalar perturbation show the amplitude of scalar perturbation given by [30]

$$
\mathscr{P}_{s}=\left(\frac{H^{2}}{2 \pi\left(c_{A}(\rho+p)\right)^{1 / 2}}\right)^{2},
$$

where $c_{A}$ is sound speed and for our model is a constant and equal to $c_{A}^{2}=(2 n-1)^{-1}$ (reader could refer to [30] for more detail).

Besides scalar fluctuation, the inflationary scenario predicts tensor fluctuation, which is known as a gravitational wave, too. The produced tensor fluctuations induce a curved polarization in the $\mathrm{CMB}$ radiation and increase the overall amplitude of their anisotropies at a large scale. The physics of the early universe could be specified by fitting the analytical results of $\mathrm{CMB}$ and density spectra to corresponding observational data. At first, it was thought that the possible effects of primordial gravitational waves are not important and might be ignored. However, a few years ago, it was found out that the tensor fluctuations have an important role, and they should be taken into consideration to determine the best-fit values of the cosmological parameters from the CMB and LSS spectra [34-36]. Contribution of tensor perturbation in metric is expressed as

$$
d s^{2}=-d t^{2}+a^{2}(t)\left(\delta_{i j}+h_{i j}\right) d x^{i} d x^{j}
$$

Inserting it into field equations comes to tensor perturbation equations. In contrast with scalar and vector perturbation, energy-momentum perturbation has no role in tensor perturbation equation. After doing some algebraic analysis, the amplitude of tensor perturbation is obtained as [30]

$$
\mathscr{P}_{T}=\frac{8}{M_{p}^{2}}\left(\frac{H}{2 \pi}\right)^{2} .
$$

The imprint of tensor fluctuation on the CMB brings this idea to indirectly determine its contribution to power spectra by measuring CMB polarization [35]. Such a contribution could be expressed by the $r$ quantity, which is known as tensor-toscalar ratio and represents the relative amplitude of tensorto-scalar fluctuation: $r=\mathscr{P}_{T} / \mathscr{P}_{s}$. Therefore, constraining $r$ is one of the main goals of the modern CMB survey. According to the current accuracy of observations, it is only possible to place a constant upper bound on the allowed range of $r$ [37-42]. Recent data from nine years of results of WMAP9 and South Pole Telescope (SPT) give the latest constraints of $r<0.13$ and $r<0.11$ at 95\% confidence level (CL) [43-46]. Combining Planck's temperature anisotropy measurements with the WMAP large-angle polarization to constrain inflation gives an upper limit $r<0.11$ in $95 \%$ CL $[17,46]$. The latest data about the quantity comes from Planck collaboration on February 2015. Planck full mission data for $\Lambda C D M+r$ model resulted in a new constraint on the quantity $r$ as $r<0.10$ (Planck TT, TE, EE + lowP), $<0.11$ (Planck TT + lowP + lensing) at 95\% CL, which indicates a slight improvement in comparison with the previous result of Planck-2013 [19, 25].

\section{Chaotic Inflation}

The general form of dynamical equations and main parameters of the model have been derived, which brings us some crude results. In order to have more clear insight, a specific kind of potential is necessary. In this section, we are going to consider the model for a familiar kind of potential, which has received lots of attention, namely, power-law potential.

Using slow-roll approximations, the dynamical equation of the model is rewritten as

$$
\begin{aligned}
H^{2} & =\frac{1}{3 M_{p}^{2}} V(\phi), \\
\dot{H} & =-\frac{1}{M_{p}^{2}}\left(n F_{0} X^{n}\right)
\end{aligned}
$$


and the acceleration equation is expressed as $\ddot{a} / a=$ $V(\phi) / 3 M_{p}^{2}$ which shows a positive acceleration, a desirable situation for inflation. Also, for the wave equation, there is

$$
3 H \dot{\phi}\left(n F_{0} X^{n-1}\right)+V^{\prime}(\phi)=0 .
$$

By utilizing the definition of slow-roll parameters, and the reorganized form of dynamical equations, the slow-roll parameters could be obtained as

$$
\begin{aligned}
& \epsilon_{V}=\frac{3 n F_{0}}{V(\phi)}\left[\frac{-M_{p} V^{\prime}(\phi)}{n F_{0} \sqrt{6 V(\phi)}}\right]^{2 n /(2 n-1)}, \\
& \eta_{V}=\frac{M_{p}^{2} V^{\prime \prime}(\phi)}{n F_{0} V(\phi)}\left[\frac{n F_{0} \sqrt{6 V(\phi)}}{-M_{p} V^{\prime}(\phi)}\right]^{2(n-1) /(2 n-1)}
\end{aligned}
$$

This form of slow-roll parameters is known as potential slow-roll parameters, whose smallness displays the flatness of potential during inflation. Inflation period lasts until the slow-roll parameter $\epsilon_{V}$ arrives at one, which corresponds to $\ddot{a}=0$. Amount of expansion during this era is measured by number of $e$-folds' parameter, indicated by $N$ and defined as

$$
\begin{aligned}
N & \equiv \ln \left(\frac{a_{e}}{a_{i}}\right)=\int_{t_{i}}^{t_{e}} H d t=\int_{\phi_{i}}^{\phi_{e}} \frac{H}{\dot{\phi}} d \phi \\
& =\left(\frac{-n F_{0}}{6^{n-1} M_{p}^{2 n}}\right)^{1 /(2 n-1)} \\
& \cdot \int_{\phi_{i}}^{\phi_{e}} \sqrt{V(\phi)}\left(\frac{\sqrt{V(\phi)}}{V^{\prime}(\phi)}\right)^{1 /(2 n-1)} .
\end{aligned}
$$

It is expressed that, to overcome on standard cosmology problems, there should be about 55-65 numbers of $e$-folds.

Let us turn our attention to power-law potential $V(\phi)=$ $\lambda \phi^{\alpha}$. Since the general form of main equations has been acquired in the previous section, we ignored repeating it, and we only expressed the final results. Substituting this potential in slow-roll parameters (13), one arrives at

$$
\begin{aligned}
\epsilon_{V} & =\frac{3 n F_{0} \mathscr{F}^{n}}{\lambda} \phi^{-z / \bar{n}}, \\
\eta_{V} & =\frac{\alpha(\alpha-1) M_{p}^{2}}{n F_{0} \mathscr{F}^{n-1}} \phi^{-z / \bar{n}}
\end{aligned}
$$

in which

$$
\begin{aligned}
\mathscr{F} & \equiv\left[\frac{\alpha^{2} M_{p}^{2} \lambda}{6 n^{2} F_{0}^{2}}\right]^{1 / \bar{n}}, \\
z & \equiv \alpha n+2 n-\alpha, \\
\bar{n} & \equiv 2 n-1 .
\end{aligned}
$$

At the end of inflation era, the acceleration parameter vanishes, and the slow-roll parameter $\epsilon_{V}$ reaches to one. Therefore, at this time, the scalar field could be read from (15) as

$$
\phi_{e}^{z / \bar{n}}=\frac{3 n F_{0} \mathscr{F}^{n}}{\lambda} .
$$

Using the number of $e$-folds (14), one could easily derive the scalar field at the beginning of inflation

$$
\phi_{i}^{z / \bar{n}}=\mathscr{A} \phi_{e}^{z / \bar{n}}
$$

in which $\mathscr{A}$ is a constant parameter, defined by $\mathscr{A} \equiv 1+$ $2 z N / \alpha \bar{n}$.

As it was mentioned, at the beginning of inflation, in order to have a quasi-de Sitter expansion, the slow-roll parameters should be much smaller than unity. Therefore, the constants should be in a way to satisfy this condition.

Using the definition of the potential in perturbation amplitude (8) and (10), the scalar and tensor perturbation amplitudes, respectively, are obtained as

$$
\begin{aligned}
\mathscr{P}_{s} & =\frac{\sqrt{\bar{n}} \lambda^{2}}{72 \pi^{2} M_{p}^{4} n F_{0} \mathscr{F}^{n}} \phi^{\alpha} \phi^{z / \bar{n}}, \\
\mathscr{P}_{T} & =\frac{2}{3 \pi^{2} M_{p}^{4}} \lambda \phi^{\alpha} \Longrightarrow r=\frac{16}{\sqrt{2 n-1}} \epsilon_{V} .
\end{aligned}
$$

The tensor perturbation is observed indirectly, by using the parameter $r$ which is the ratio of tensor perturbation amplitude to scalar perturbation amplitude: $r=\mathscr{P}_{T} / \mathscr{P}_{s}$. These perturbations are used to constrain another free parameter of the model; however first we need to compute them at initial of inflation, namely, for $\phi=\phi_{i}$.

On the other hand, the scalar and tensor spectra indices are expressed in terms of these slow-roll parameters

$$
\begin{aligned}
n_{s}-1 & =\frac{d \ln \left(\mathscr{P}_{s}\right)}{d \ln (k)}=\frac{2 n}{2 n-1} \eta_{V}-\frac{2(5 n-2)}{2 n-1} \epsilon_{V}, \\
n_{T} & =\frac{d \ln \left(\mathscr{P}_{s}\right)}{d \ln (k)}=-2 \epsilon_{V} .
\end{aligned}
$$

Utilizing the scalar spectra index (20), and tensor-toscalar ratio equations (19), one could determine the behavior of the parameter $n$ in terms of $n_{s}$ and $r$. Based on latest Planck data, there are a range for scalar spectra index and an upper bound for tensor-to-scalar ratio [19]:

$$
\begin{aligned}
& * \mathrm{TT}+\text { lowP } \rightarrow n_{s}=0.9655 \pm 0.0062, r<0.10 . \\
& * \mathrm{TT}+\text { lowP }+ \text { lensing } \rightarrow n_{s}=0.9677 \pm 0.0060, r< \\
& 0.11 \text {. } \\
& * \mathrm{TT}+\text { lowP }+\mathrm{BAO} \rightarrow n_{s}=0.9673 \pm 0.0045, r< \\
& 0.11 \text {. } \\
& * \mathrm{TT}, \mathrm{TE}, \mathrm{EE}+\text { lowP } \rightarrow n_{s}=0.9645 \pm 0.0049, r< \\
& 0.10 .
\end{aligned}
$$

Note that, as we concentrate on Planck-2015 data about the quantity $r$, we realize that the previous mentioned 


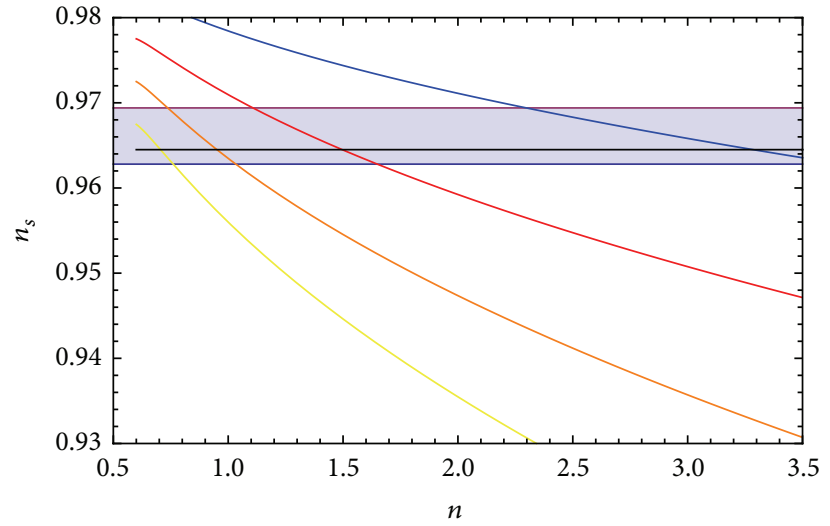

(a) $n_{s}-n$

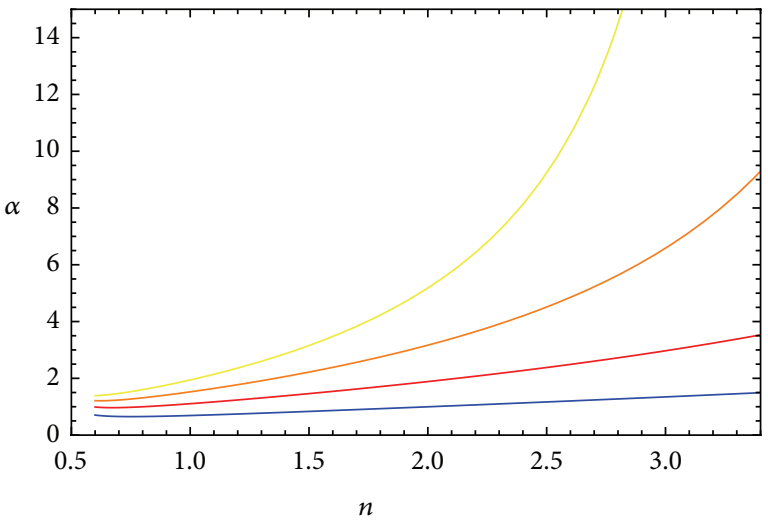

(b) $\alpha-n$

FIgURE 1: (a) The scalar spectra index versus $n$. (b) The potential power parameter $\alpha$ is depicted versus $n$; for four different values of tensorto-scalar ratio as $r=0.05$ (blue line), $r=0.09$ (red line), $r=0.11$ (orange line), and $r=0.14$ (yellow line).

TABLE 1: The final results for the parameters $n$ and $\alpha$.

\begin{tabular}{lllll}
\hline$r$ & 0.05 & 0.08 & 0.11 & 0.14 \\
\hline$n$ & 3.28 & 1.50 & 0.95 & 0.70 \\
$\alpha$ & 1.45 & 1.46 & 1.46 & 1.47 \\
\hline
\end{tabular}

constraint could rise in some cases. For instance, according to [19], for $\Lambda \mathrm{CDM}+r+d \ln n_{s} / d \ln k$ model, there is $r<0.176$ (Planck TT + lowP + lensing).

In order to specify a proper value for the constant parameter $n$, the parameter is depicted versus $n_{s}$ for four different values of $r$ in Figure 1(a). The shadow covers the area which stays in the range $0.9628<n_{s}<0.9694$; the common interval for scalar spectra index resulted from Planck-2015 data. The curves cross the shadow line, which in turn indicates interval values of $n$ which are in perfect match with observational data. Figure 1(b) displays the parameter $\alpha$ in terms of $n$ for four different values of tensor-to-scalar ratio. In return to the interval values of $n$ that comes from Figure 1(a), one could demonstrate appropriate values for the potential power $\alpha$. The final result has been prepared in Table 1. The existence of a modified kinetic term for scalar field is clear from the result, in which the power of kinetic term runs from 0.70 to 3.28 . In addition, the appropriate potential, to be in agreement with observational data, has a power of $\alpha \simeq 1.46$.

Finally, Figure 2 illustrates the tensor-to-scalar ratio in term of scalar spectra index for the determined values of the parameters $n$ and $\alpha$. Compatability of $r$ behavior states that the model parameters are chosen properly.

\section{Hamilton-Jacobi Approach}

The Hamilton-Jacobi formalism is utilized as a strong tool to provide numerous inflationary models with exactly known analytic solutions for the background expansion. One of the most interesting advantages of the formalism is that almost the whole parameters of the model could be derived that

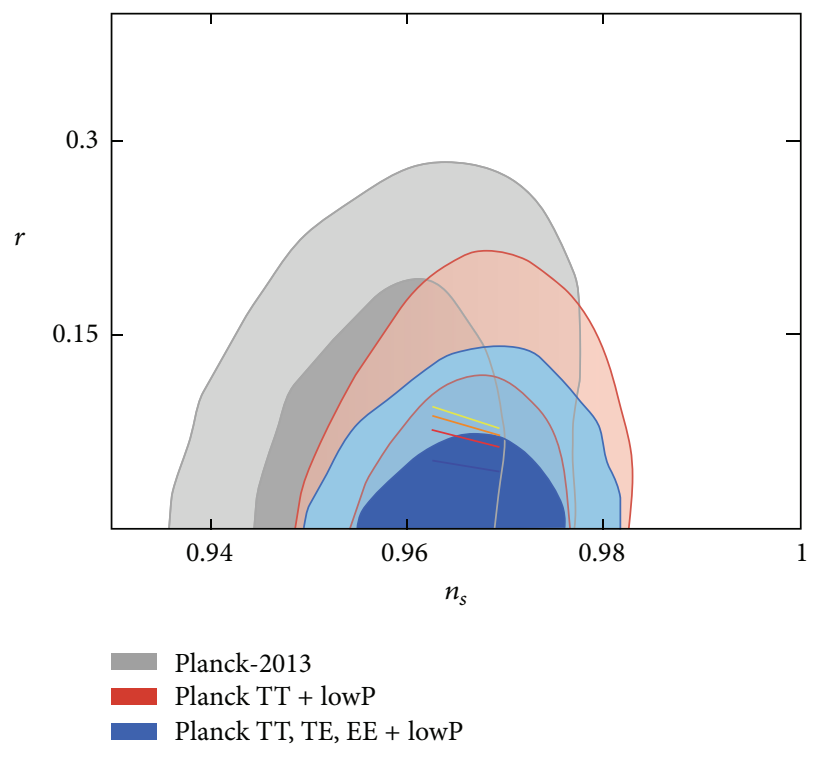

FIgURE 2: The figure shows the tensor-to-scalar ratio versus the scalar spectra index.

is explained in the following lines [20-24]. Suppose the Hubble parameter could be defined as a function of scalar field $H:=H(\phi)$. Consequently, the time derivative of the Hubble parameter is reexpressed as $\dot{H}=\dot{\phi} H^{\prime}$, where prime denotes derivative with respect to scalar field. Then, the second Friedmann equation is read as the following:

$$
\dot{\phi}^{\bar{n}}=-\frac{2^{n} M_{p}^{2}}{n F_{0}} H^{\prime}
$$

where the prime is derivation with respect to scalar field. Taking integrate of the above relation produces a scalar field in terms of scalar field, and in turn the Hubble parameter could be displayed in terms of time as well. Eventually, 
the model prediction about the potential is achievable by substituting (21) in the Friedmann equation (2)

$$
V(\phi)=3 M_{p}^{2} H^{2}(\phi)-\frac{\bar{n} F_{0}}{2^{n}}\left(-\frac{2^{n} M_{p}^{2}}{n F_{0}} H^{\prime}\right)^{2 n / \bar{n}} .
$$

It could be named as the most important consequence of the formalism, so that, for any specific function of $H(\phi)$, the model produces a potential $V(\phi)$ which admits the given $H(\phi)$ as an exact inflationary solution [20, 47]. Equations (21) and (22) are known as Hamilton-Jacobi equations.

The slow-roll parameters of the model are expressed in terms of Hubble parameter and its derivatives, in which for the tow well-known parameters we have

$$
\begin{aligned}
& \epsilon_{H}=-\left(-\frac{2^{n} M_{p}^{2}}{n F_{0}}\right)^{1 / \bar{n}} \frac{H^{\prime 2 n / \bar{n}}}{H^{2}} \\
& \eta_{H}=\frac{-1}{\bar{n}}\left(-\frac{2^{n} M_{p}^{2}}{n F_{0}}\right)^{1 / \bar{n}} \frac{H^{\prime 2(1-n) / \bar{n}} H^{\prime \prime}}{H} .
\end{aligned}
$$

Bringing out the same argument as we had in the previous case, one could read the scalar field at the end of inflation. The initial value of scalar field could be derived from number of $e$-folds' relation, so that we have

$$
N=\int_{\phi_{i}}^{\phi_{e}} \frac{H}{\dot{\phi}} d \phi=\int_{\phi_{i}}^{\phi_{e}}\left(-\frac{n F_{0}}{2^{n} M_{p}^{2}}\right)^{1 / \bar{n}} \frac{H}{H^{\prime 1 / \bar{n}}} d \phi .
$$

The general form of amplitude of scalar and tensor quantum perturbations were introduced, respectively, in (12) and (14). It was explained how the observational quantities $n_{s}$ and $n_{T}$ could result from the corresponding perturbation amplitude. Then, the scalar and tensor spectra indices are given by

$$
\begin{gathered}
n_{s}-1=2 n \eta_{H}-4 \epsilon_{H} ; \\
n_{T}=-2 \epsilon_{H} .
\end{gathered}
$$

All required equations for considering inflation era using Hamilton-Jacobi approach were introduced so far. The next step for going ahead and studying the situation in more detail is to pick out a specific function for the Hubble parameter in terms of scalar field. Then, it is assumed that the Hubble parameter could be expressed as a power-law function of scalar field, so that $H(\phi)=H_{0} \phi^{\beta}$.

Substituting the expression of the Hubble parameter in the slow-roll equations (23) comes to the following consequences:

$$
\begin{aligned}
& \epsilon_{H}=-\left(-\frac{2^{n} M_{p}^{2}}{n F_{0}}\right)^{1 / \bar{n}} \frac{\beta^{2 n / \bar{n}}}{H_{0}^{2(n-1) / \bar{n}}} \phi^{\sigma / \bar{n}} \\
& \eta_{H}=-\frac{(\beta-1) \beta^{1 / \bar{n}}}{\bar{n}}\left(-\frac{2^{n} M_{p}^{2}}{n F_{0}}\right)^{1 / \bar{n}} H_{0}^{2(1-n) / \bar{n}} \phi^{\sigma / \bar{n}}
\end{aligned}
$$

where the constant parameter $\sigma$ is defined as $\sigma=2 \beta-$ $2 n \beta-2 n$. The inflation ends when the slow-roll parameter

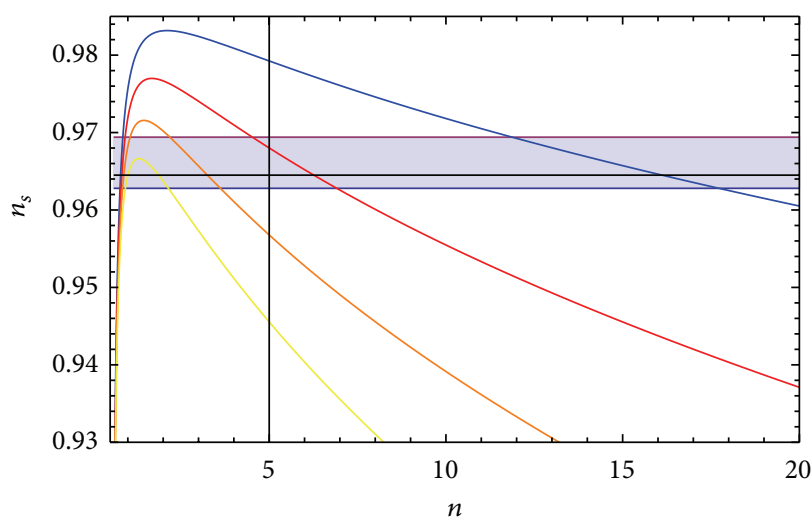

FIgURE 3: The scalar spectra index versus $n$ for four different values of tensor-to-scalar ratio, as $r=0.05$ (blue line), $r=0.09$ (red line), $r=0.11$ (orange line), and $r=0.14$ (yellow line).

$\epsilon_{H}$ reaches unity, which in turn describes $\ddot{a}=0$, and the final scalar field could be read easily. As it was mentioned, the scalar field at the beginning of inflation is acquired from number of $e$-folds' relation as well. Final and initial scalar field, respectively, resulted as

$$
\begin{aligned}
\phi_{e}^{\sigma / \bar{n}} & =-\left(-\frac{n F_{0}}{2^{n} M_{p}^{2}}\right)^{1 / \bar{n}} \frac{H_{0}^{2(n-1) / \bar{n}}}{\beta^{2 n / \bar{n}}} \\
\phi_{i}^{\sigma / \bar{n}} & =\frac{1}{\mathscr{B}} \phi_{e}^{\sigma / \bar{n}},
\end{aligned}
$$

where the redefined constant $\mathscr{B}$ is given by $\mathscr{B}=1-\sigma N / \beta$.

Consequently, the perturbation quantities could be derived at the beginning of inflation by inserting the initial value of scalar field in the corresponding relations. Therefore, the spectra indices are described as

$$
\begin{aligned}
n_{s}-1 & =\frac{4 \beta-6 n \beta-2 n}{\bar{n} \beta \mathscr{B}} ; \\
n_{T} & =-\frac{2}{\mathscr{B}},
\end{aligned}
$$

and the tensor-to-scalar ratio, which indirectly indicates the presence of gravitational waves, comes to the following expression:

$$
\begin{aligned}
r & =\frac{16 c_{A}^{2}}{\mathscr{B}} \\
\text { or } r & =\frac{16 c_{A}^{2} \bar{n} \beta}{4 \beta-6 n \beta-2 n}\left(n_{s}-1\right) .
\end{aligned}
$$

Utilizing the relations for $n_{s}, r$ and following the same process as previous section, the behavior of the constant parameter $n$ could be specified easily in terms of $n_{s}$ and $r$. Figure 3 portrays the behavior versus scalar spectra index for four different values of $r$. In comparison with the previous method, there are two separate intervals for $n$ which could be consistent with observational data. 


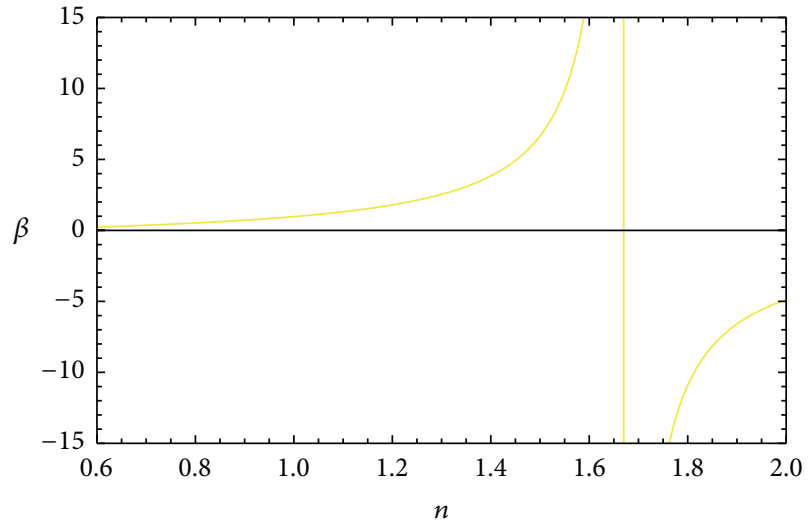

(a) $r=0.08$

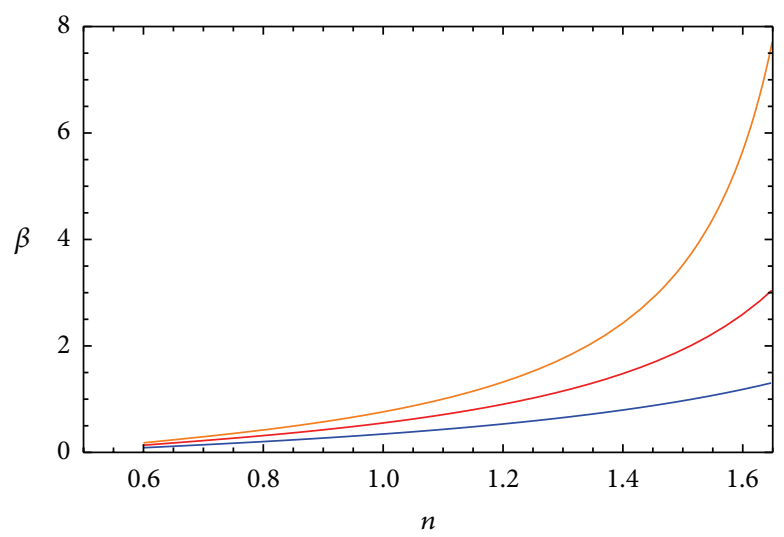

(b) $r=0.08$

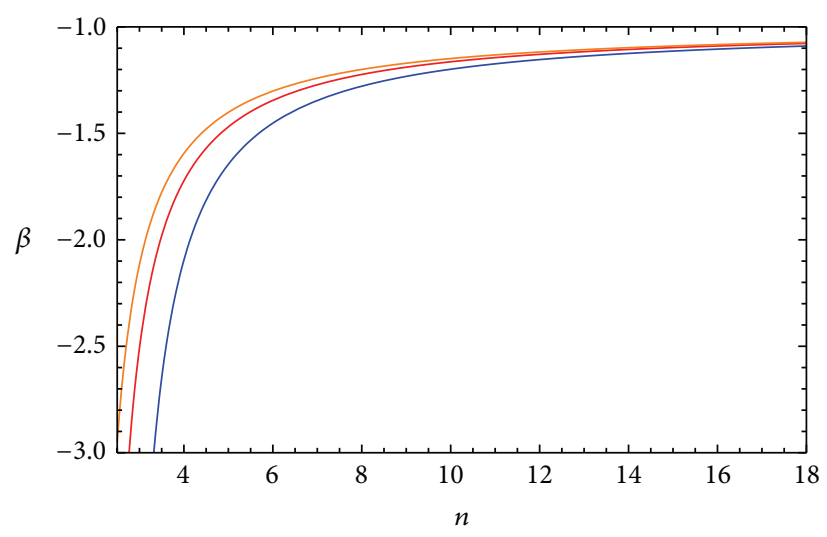

(c) $r=0.10$

Figure 4: The Hubble power parameter $\beta$ is depicted versus $n$ for four different values of tensor-to-scalar ratio, as $r=0.05$ (blue line), $r=0.08$ (red line), $r=0.11$ (orange line), and $r=0.14$ (yellow line).

TABLE 2: The final results for the parameters $n$ and $\alpha$.

\begin{tabular}{lcccc}
\hline$r$ & 0.05 & 0.08 & 0.11 & 0.14 \\
\hline$n_{1}$ & 0.80 & 0.83 & 0.88 & 1.00 \\
$\beta_{1}$ & 0.20 & 0.34 & 0.54 & 0.97 \\
\hline$n_{2}$ & 16.12 & 6.26 & 3.25 & 1.86 \\
$\beta_{2}$ & -1.10 & -1.32 & -1.92 & -7.77 \\
\hline
\end{tabular}

Definitely, for these intervals of $n$, there are two intervals for the Hubble power parameter $\beta$. The behavior of the parameter versus $n$ is illustrated in Figure 4 for four different values of $r$. Figure 4(a) is plotted for $r=0.05$. Figures 4(b) and 4(c) depict the behavior of $\beta$ versus $n$ to identify proper values of $\beta$, respectively, for the first and second interval of $n$. Ultimate result has been expressed in Table 2 .

Hamilton-Jacobi formalism again indicates the necessity of modified version of kinetic term for scalar field; however, the difference is that the power of kinetic term sounds smaller than unity for $n_{1}$, about $n_{1} \simeq 0.8$, and larger than previous case for $n_{2}$, about $n_{2} \simeq 0.8$. The result for power of Hubble parameter displays that it could be both positive for $n_{1}$ and negative for $n_{2}$. The whole result has been presented in Table 2.
So far, the constant parameters $n$ and $\beta$ have been determined by using observational data. Then, these results could be applied in (30), to consider the behavior of $r$ in terms of $n_{s}$, as shown in Figure 5 for two separate sets of $(n, \beta)$. Suitability of $r$ behavior and standing in the right region prove that the free parameters have been chosen properly.

4.1. Potential. The general form of the potential was introduced in (22), and it is a polynomial in $\phi$. In order to study the behavior of the potential during the inflationary times, the potential could be drawn in terms of scalar field using the constant parameters $n$ and $\beta$ from Table 2 . It has been depicted for four different values of tensor-to-scalar ration, displayed in Figure 6. In all cases, the potential reduces with increasing time (or decreasing scalar field), and the only difference stands in the slip of the potential curve. The potential diagram is convex, and with increasing $r$, it alters and turns to a convex diagram. For $r=0.11$, it looks like a linear potential when it behaves as $V \propto \phi$; and for $r=0.14$, it behaves like the well-known and common potential $V \propto$ $\phi^{2}$. Note that, during inflationary times, the initial and final values of the scalar field are smaller than Planck mass in the first three cases; and in the final plot (where $r=0.14$ ), only the initial values of scalar field become larger than Planck mass. 


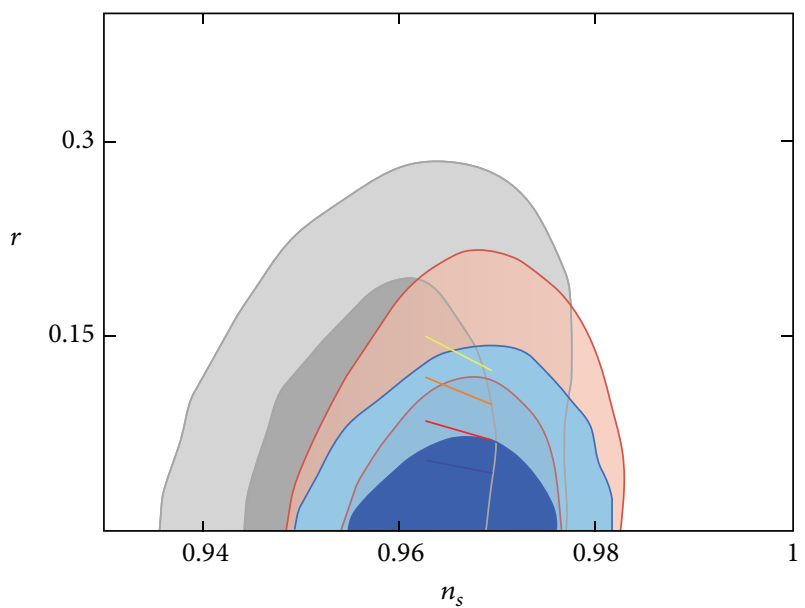

Planck-2013

Planck TT + lowP

Planck TT, TE, EE + lowP

(a) $r=0.08$

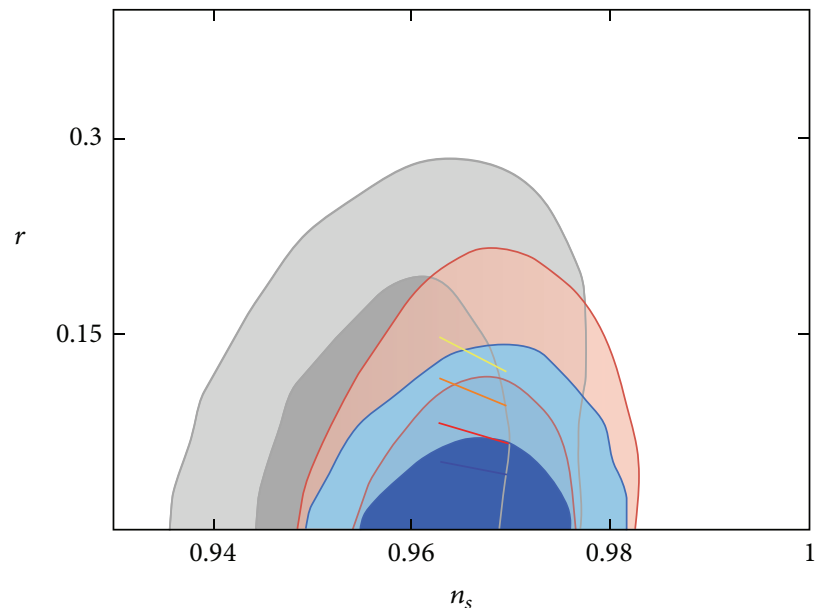

Planck-2013

Planck TT + lowP

Planck TT, TE, EE + lowP

(b) $r=0.10$

FIGURE 5: The figure shows the tensor-to-scalar ratio versus the scalar spectra index.
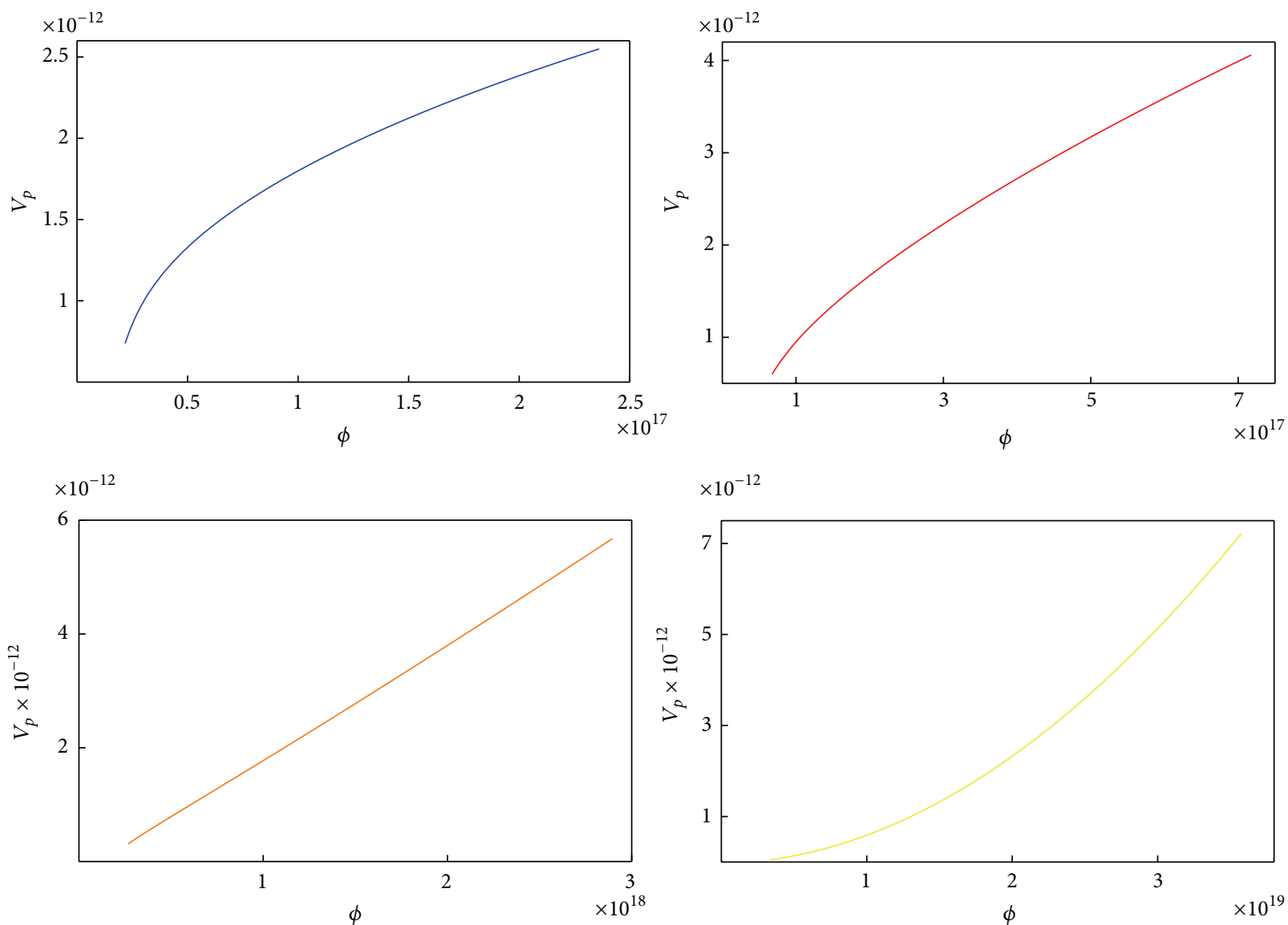

FiguRE 6: The potential behavior has been portrayed for different values of tensor-to-scalar ratio as: $r=0.05$ (blue), $r=0.08$ (red), $r=0.11$ (orange), and $r=0.14$ (yellow). 
Then, it results that the inflation could occur for field values below the Planck mass; almost the same result was obtained in [48], where the authors study chaotic inflation in braneworld scenario. In addition, as it was expected, the potential energy density is always smaller than Planck energy.

4.2. Attractive Behavior. Following [49], the attractive behavior of the model could be investigated by adding a homogeneous perturbation $\delta H(\phi)$ to a hypothetical solution $H_{0}(\phi)$ of Hamilton-Jacobi equation (22). If the expression $\delta H(\phi) / H_{0}(\phi)$ approaches to zero by passing time, the attractor condition could be satisfied. Substituting $H(\phi)=H_{0}(\phi)+$ $\delta H(\phi)$ in the Hamilton-Jacobi equation and linearizing it, the perturbation equation is read as

$$
H_{0}^{\prime 1 / \bar{n}} \delta H^{\prime}=-\frac{3 \bar{n}}{2 n}\left(-\frac{n F_{0}}{2^{n} M_{p}^{2}}\right)^{1 / \bar{n}} H_{0} \delta H .
$$

Taking integrate of above equation, one arrives at the following expression for the perturbation $\delta H$ :

$$
\delta H=\delta H\left(\phi_{i}\right) \exp \left(-\frac{3 \bar{n}}{2 n} N\right) .
$$

Equation (32) strongly points out the fact that, by passing time, the perturbation parameter $\delta H$ vanishes, and the model successfully satisfies the attractor condition.

\section{Special Case}

The kinetic term of scalar field was taken as a power-law function of $X$; namely, $F(X)=F_{0} X^{n}$. For such a kinetic energy, one is faced with a special case when the power of the term is taken as $n=1 / 2$. In this section, we are going to briefly review the case.

Substituting $n=1 / 2$ in the general dynamical equations obtained in Section 2 comes to the following result for Friedmann equations:

$$
\begin{aligned}
H^{2} & =\frac{1}{3 M_{p}^{2}} V(\phi) \\
\dot{H} & =-\frac{F_{0}}{2 M_{p}^{2}} \sqrt{X} .
\end{aligned}
$$

The first surprising conclusion is that the energy density of scalar field is exhibited by potential energy density, and the kinetic term has no contribution. Also, taking integrate of (34) gives the Hubble parameter in terms of scalar field,

$$
H(\phi)=\frac{\mp F_{0}}{2 \sqrt{2} M_{p}^{2}} \phi+c_{1},
$$

where $c_{1}$ is a constant of integration and $\mp$, respectively, are related to $\sqrt{X} \propto \dot{\phi}$ and $\sqrt{X} \propto-\dot{\phi}$. In addition to the Hubble parameter, the potential energy density could be derived exactly from the equation of motion of scalar field, so that

$$
\frac{3 F_{0}}{2} H \frac{\sqrt{2}}{\dot{\phi}} \phi+V^{\prime}(\phi)=0,
$$

and by taking integrate and utilizing the first Friedmann equation (33), the potential is expressed by

$$
V(\phi)=\frac{1}{4}\left(\frac{\mp 3 F_{0}}{\sqrt{6} M_{p}} \phi+c_{2}\right)^{2},
$$

in which $c_{2}$ is constant of integration, related to $c_{1}$ by $c_{2}=$ $2 M_{p} \sqrt{3} c_{1}$. Note that, by rescaling the scalar field, the potential could be rewritten as a potential of massive scalar field $V(\varphi)=m_{\varphi}^{2} \varphi^{2} / 2$, so that $\varphi \equiv \mp \phi+2 \sqrt{2} M_{p}^{2} c_{1} / F_{0}$, and $m_{\varphi} \equiv$ $\sqrt{3 / 4} F_{0} / M_{p}$.

As a further argument, it should be mentioned that the dynamical equations of this special case are automatically in the form of the dynamical equation of usual cases after applying the slow-rolling approximation.

\section{Conclusion}

The inflationary scenario was studied using a noncanonical scalar field instead of usual canonical scalar field. It was supposed that the kinetic term, $F(X)$, is a power-law function of $X\left(=\nabla_{\mu} \phi \nabla^{\mu} \phi / 2\right)$; namely, there is $F(X)=F_{0} X^{n}$, where $F_{0}$ is a constant parameter. In the slow-roll inflationary scenario, regardless of whether scalar field is canonical or noncanonical, the time rate of the Hubble parameter during a Hubble time should be smaller than unity, and the same behavior is supposed for time derivative of scalar field, known as slow-roll approximation. The work was implemented by using two formalisms, and the main goal was to constrain the free parameter of the model coming from the latest observational data.

Recent observational data gives us a proper insight about some perturbation parameters such as spectra indices and amplitude of perturbations. The latest data is related to Planck, released on 2015. The result exhibits a slight correction to Planck-2013 and states that the scalar spectra index is about $n_{s}=0.9645 \pm 0.0049$, and the amplitude of the perturbation is measured as $\ln \left(10^{10} \mathscr{P}_{s}\right)=3.094$. The tensor perturbation is still not clear as the scalar perturbation so that we only have an upper bound for tensor-to-scalar ratio as $r<0.10$.

To start, the first formalism was used and a general form of a power-law potential was proposed to consider the situation in detail. After obtaining the general form of evolution equations, and using slow-roll approximations, the slow-roll parameters $\epsilon$ and $\eta$ were derived for noncanonical scalar field model including a power-law kinetic term. As it was expected, these parameters come back to the standard form of slow-roll parameters for $n=F_{0}=1$. Utilizing the Planck data, we determined the power of kinetic term and potential, namely, $n$ and $\alpha$, presented in Table 1 . The power of kinetic energy term is obtained as $n \approx 0.70,1.5,3$ which indicates the necessity of a noncanonical term for kinetic energy of scalar field. In addition, the constraint values for power of potential function showed that this parameter should be about $\alpha \approx 1.46$ which are in good agreement with the recent observational results about the form of potential in inflationary era. 
At next step, the Hamilton-Jacobi formalism was applied by presenting a power-law function of scalar field for the Hubble parameter, $H \propto \phi^{\beta}$. Repeating the same process resulted in a noncanonical kinetic term for scalar field so that the parameter $n$ changes from 0.80 to 1.00 ; and the power of the Hubble parameter $\beta$ stands in the range $0.20-0.97$. The constraint values were utilized to portray the predicted potential of the model. By contrast to inflationary studies using the first formalism, considering the potential behavior points out that the inflation could occur even when the field values stand below the Planck mass. During inflation times, the potential decreases with reduction of scalar field.

\section{Conflict of Interests}

The authors declare that there is no conflict of interests regarding the publication of this paper.

\section{References}

[1] A. D. Linde, Particle Physics and Inationary Cosmology, Harward Academic, Chur, Switzerland, 1990.

[2] A. H. Guth, "Inflationary universe: a possible solution to the horizon and flatness problems," Physical Review D, vol. 23, pp. 347-356, 1981.

[3] A. Linde, "Chaotic inflation," Physics Letters B, vol. 129, no. 3-4, pp. 177-181, 1983.

[4] A. Melchiorri, L. Mersini, C. J. Ödman, and M. Trodden, “The state of the dark energy equation of state," Physical Review D, vol. 68, no. 4, Article ID 043509, 2003.

[5] A. Sen, "Field theory of Tachyon matter," Modern Physics Letters A, vol. 17, no. 27 , p. 1797, 2002.

[6] N. D. Lambert and I. Sachs, "Tachyon dynamics and the effective action approximation," Physical Review D, vol. 67, no. 2, Article ID 026005, 8 pages, 2003.

[7] T. Chiba, T. Okabe, and M. Yamaguchi, "Kinetically driven quintessence," Physical Review D, vol. 62, no. 2, Article ID 023511, 8 pages, 2000.

[8] C. Armendariz-Picon, V. Mukhanov, and P. J. Steinhardt, "Dynamical solution to the problem of a small cosmological constant and late-time cosmic acceleration," Physical Review Letters, vol. 85, no. 21, pp. 4438-4441, 2000.

[9] C. Armendariz-Picon and V. Mukhanov, "Essentials of kessence," Physical Review D, vol. 63, no. 10, Article ID 103510, 2001.

[10] T. Chiba, “Tracking k-essence," Physical Review D, vol. 66, Article ID 063514, 2002.

[11] L. P. Chimento and A. Feinstein, "Power-low expansion in kessence cosmology," Modern Physics Letters A, vol. 19, no. 10, pp. 761-768, 2004.

[12] L. P. Chimento, "Extended tachyon field, Chaplygin gas, and solvable $k$-essence cosmologies," Physical Review D, vol. 69, no. 12, Article ID 123517, 2004.

[13] C. Armendáriz-Picón, T. Damour, and V. Mukhanov, " $k$-inflation," Physics Letters B, vol. 458, no. 2-3, pp. 209-218, 1999.

[14] J. Garriga and V. F. Mukhanov, "Perturbations in $k$-inflation," Physics Letters B, vol. 458, no. 2-3, pp. 219-225, 1999.

[15] W. Fang, H. Q. Lu, and Z. G. Huang, "Cosmologies with a general non-canonical scalar field," Classical and Quantum Gravity, vol. 24, no. 15, pp. 3799-3811, 2007.
[16] N. Bose and A. S. Majumdar, " $k$-essence model of inflation, dark matter, and dark energy," Physical Review D, vol. 79, Article ID 103517, 2009.

[17] P. A. R. Ade, N. Aghanim, M. I. R. Alves et al., "Planck 2013 results. I. Overview of products and scientific results," Astronomy \& Astrophysics, vol. 571, article A1, 48 pages, 2014.

[18] P. A. R. Ade, N. Aghanim, C. Armitage-Caplan et al., "Planck 2013 results. XXII. Constraints on inflation," Astronomy \& Astrophysics, vol. 571, article A22, 42 pages, 2014.

[19] P. A. R. Ade, N. Aghanim, M. Arnaud et al., "Planck 2015 results. XX. Constraints on inflation," http://arxiv.org/abs/1502.02114.

[20] D. Baumann, “TASI lectures on inflation," http://arxiv.org/abs/ 0907.5424 .

[21] D. S. Salopek and J. R. Bond, "Nonlinear evolution of longwavelength metric fluctuations in inflationary models," Physical Review D, vol. 42, no. 12, pp. 3936-3962, 1990.

[22] A. G. Muslimov, "On the scalar field dynamics in a spatially flat Friedmann universe," Classical and Quantum Gravity, vol. 7, no. 2, pp. 231-237, 1990.

[23] J. E. Lidsey, "The scalar field as dynamical variable in inflation," Physics Letters B, vol. 273, no. 1-2, pp. 42-46, 1991.

[24] J. E. Lidsey, A. R. Liddle, E. W. Kolb, E. J. Copeland, T. Barreiro, and M. Abney, "Reconstructing the inflaton potential-an overview," Reviews of Modern Physics, vol. 69, no. 2, pp. 373-410, 1997.

[25] P. A. R. Ade, N. Aghanim, M. Arnaud et al., "Planck 2015 results. XIII. Cosmological parameters," http://arxiv.org/abs/ 1502.01589 .

[26] J. M. Bardeen, P. J. Steinhardt, and M. S. Turner, "Spontaneous creation of almost scale-free density perturbations in an inflationary universe," Physical Review D, vol. 28, no. 4, pp. 679-693, 1983.

[27] V. F. Mukhanov, H. A. Feldman, and R. H. Brandenberger, "Theory of cosmological perturbations," Physics Reports A: Review Section of Physics Letters, vol. 215, no. 5-6, pp. 203-333, 1992.

[28] K. A. Malik and D. Wands, "Cosmological perturbations," Physics Reports A, vol. 475, no. 1-4, pp. 1-51, 2009.

[29] S. Weinberg, Cosmology, Oxford University Press, New York, NY, USA, 2008.

[30] S. Unnikrishnan, V. Sahni, and A. Toporensky, "Refining inflation using non-canonical scalars," Journal of Cosmology and Astroparticle Physics, vol. 2012, no. 8, article no. 018, 2012.

[31] A. Riotto, "Inflation and the theory of cosmological perturbations," ICTP Summer School on Astroparticle Physics and Cosmology, Trieste, Italy, http://arxiv.org/abs/hep-ph/0210162.

[32] J. M. Bardeen, "Cosmological perturbations from quantum fluctuations to large scale structure," in Cosmology and Particle Physics. Proceedings, CCAST (World Laboratory) Symposium/Workshop, Nanjing, P.R. China, June 30-July 12, 1988, L. Fang and A. Zee, Eds., pp. 1-64, Gordon and Breach, New York, NY, USA, 1988.

[33] J.-C. Hwang, "Perturbations of the Robertson-Walker space: multicomponent sources and generalized gravity," The Astrophysical Journal, vol. 375, no. 2, pp. 443-462, 1991.

[34] W. Hu and S. Dodelson, "Cosmic microwave background anisotropies," Annual Review of Astronomy and Astrophysics, vol. 40, pp. 171-216, 2002.

[35] C. A. Terrero-Escalante, "Tensor to scalar ratio of perturbation amplitudes and inflaton dynamics," Physics Letters B, vol. 563, no. 1-2, pp. 15-22, 2003. 
[36] C. A. Terrero-Escalante, "Is power-law inflation really attractive?" Revista Mexicana de Física, vol. 49, supplement 2, pp. 118121, 2013.

[37] M. Tegmark, "Cosmological constraints from current cosmic microwave background and type IA supernova data: a brute force, eight-parameter analysis," The Astrophysical Journal, vol. 514, no. 2, pp. L69-L72, 1999.

[38] M. Tegmark, M. Zaldarriaga, and A. J. S. Hamilton, “Towards a refined cosmic concordance model: joint 11-parameter constraints from the cosmic microwave background and large-scale structure," Physical Review D, vol. 63, no. 4, Article ID 043007, 14 pages, 2001.

[39] X. Wang, M. Tegmark, and M. Zaldarriaga, "Is cosmology consistent?” Physical Review D, vol. 65, no. 12, Article ID 123001, 2002.

[40] G. Efstathiou and J. R. Bond, "Cosmic confusion: degeneracies among cosmological parameters derived from measurements of microwave background anisotropies," Monthly Notices of the Royal Astronomical Society, vol. 304, no. 1, pp. 75-97, 1999.

[41] G. Efstathiou, "Principal-component analysis of the cosmic microwave background anisotropies: revealing the tensor degeneracy," Monthly Notices of the Royal Astronomical Society, vol. 332, no. 1, pp. 193-198, 2002.

[42] G. Efstathiou, S. Moody, J. A. Peacock et al., "Evidence for a nonzero $\Lambda$ and a low matter density from a combined analysis of the $2 \mathrm{dF}$ Galaxy Redshift Survey and cosmic microwave background anisotropies," Monthly Notices of the Royal Astronomical Society, vol. 330, no. 2, pp. L29-L35, 2002.

[43] K. T. Story, C. Reichardt, Z. Hou et al., "A measurement of the cosmic microwave background damping tail from the 2500square-degree SPT-SZ survey," The Astrophysical Journal, vol. 779, article 86, 2013.

[44] G. Hinshaw, D. Larson, E. Komatsu et al., "Nine-year Wilkinson Microwave Anisotropy Probe (WMAP) observations: cosmological parameter results," The Astrophysical Journal Supplement Series, vol. 208, no. 2, p. 19, 2013.

[45] C. L. Bennett, D. Larson, J. L. Weiland et al., "Nine-year Wilkinson Microwave Anisotropy Probe (WMAP) observations: final maps and results," The Astrophysical Journal Supplement Series, vol. 208, no. 2, p. 20, 2013.

[46] K. Lau, J. Tang, and M.-C. Chu, "Cosmic microwave background constraints on the tensor-to-scalar ratio," Research in Astronomy and Astrophysics, vol. 14, no. 6, pp. 635-647, 2014.

[47] S. del Campo, "Approach to exact inflation in modified Friedmann equation," Journal of Cosmology and Astroparticle Physics, vol. 2012, no. 12, article 005, 2012.

[48] R. Maartens, D. Wands, B. A. Bassett, and I. P. Heard, "Chaotic inflation on the brane," Physical Review D, vol. 62, no. 4, Article ID 041301, 2000.

[49] D. H. Lyth and A. R. Liddle, The Primordial Density Perturbation, Cambridge University Press, Cambridge, UK, 2009. 

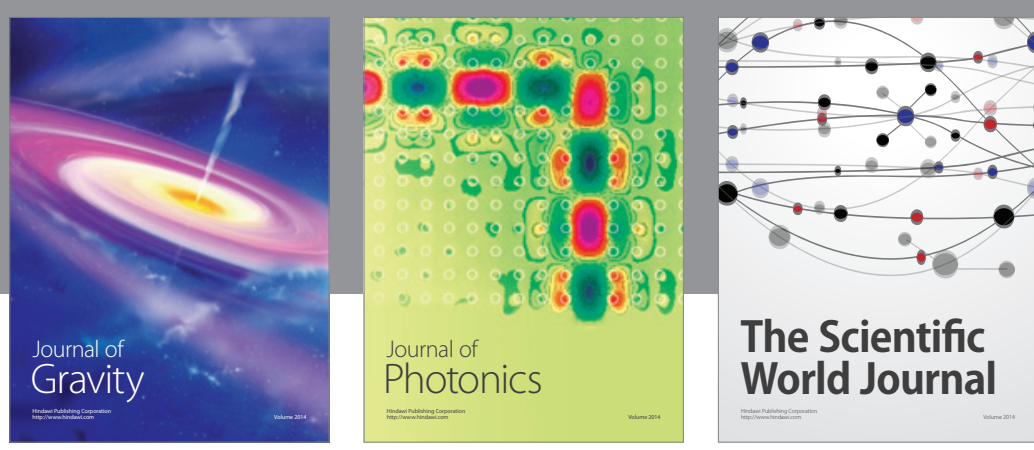

The Scientific World Journal
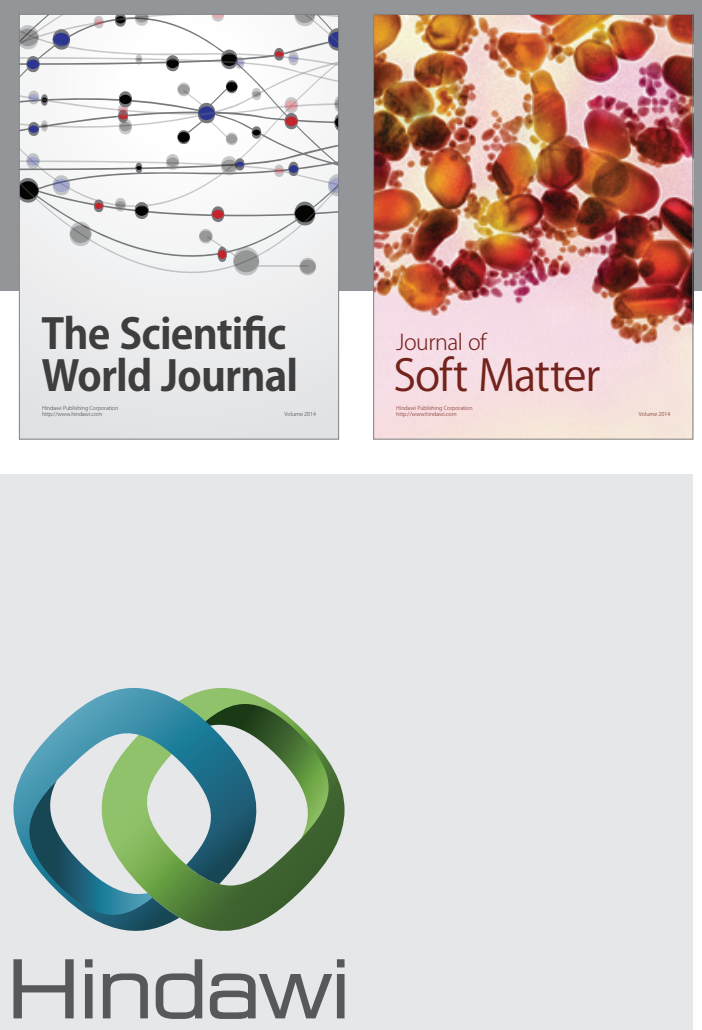

Submit your manuscripts at

http://www.hindawi.com

nternational Journal of

Statistical Mechanics
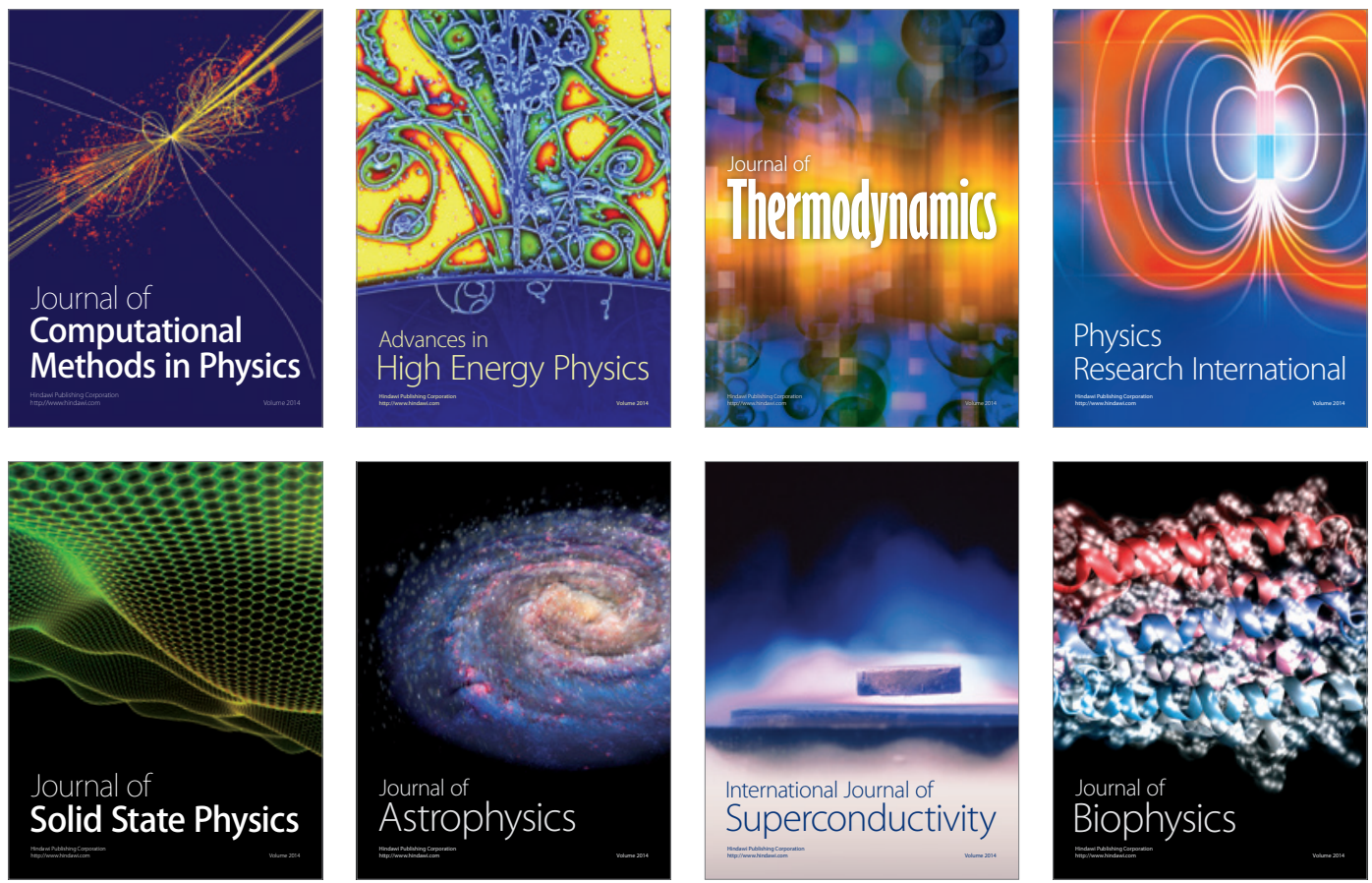
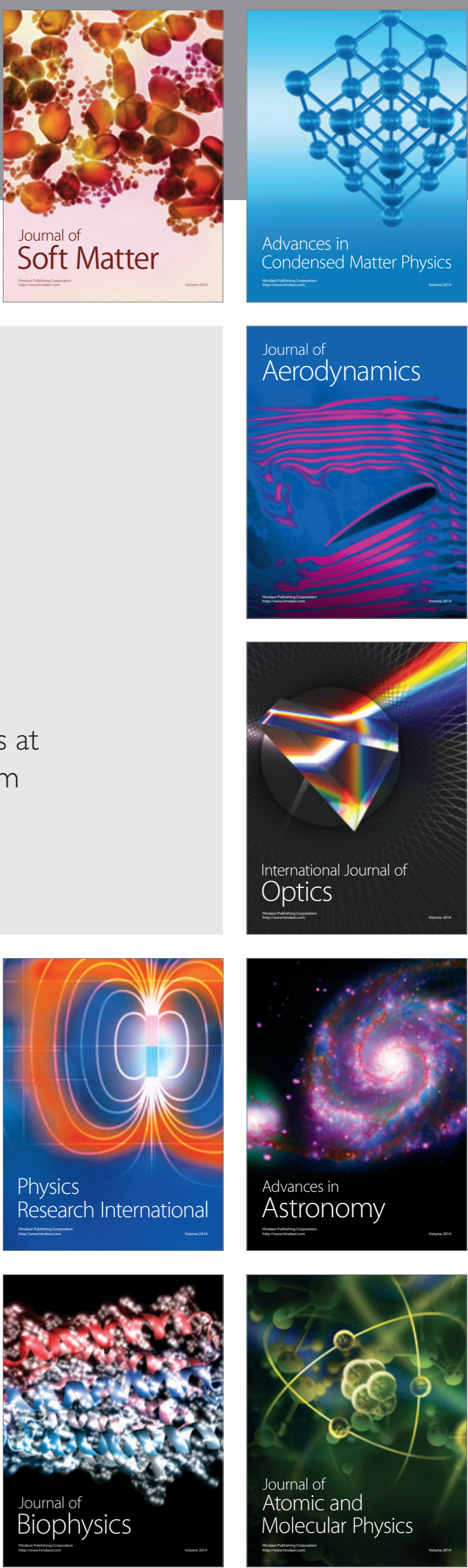